\title{
Fístula da Artéria Coronária: Associação entre Padrões de Trajetos, Características Clínicas e Cardiopatias Congênitas
}

\author{
Coronary Artery Fistula: Association between Pathway Patterns, Clinical Features and Congenital Heart Disease
}

Daniel L. Cobo, ${ }^{10}$ Fernando Batigalia, ${ }^{1}$ Ulisses A. Croti, ${ }^{1}$ Adilia M.P. Sciarra, ${ }^{1}$ Marcos H.D. Foss, ${ }^{1}$ Rafaela G.F. Cobo ${ }^{2}$

Faculdade de Medicina de São Jose do Rio Preto, ${ }^{1}$ São José do Rio Preto, SP - Brasil

Hospital da Criança e Maternidade de São José do Rio Preto, ${ }^{2}$ São José do Rio Preto, SP - Brasil

\section{Resumo}

Fundamento: A fístula da artéria coronária (FAC) é uma conexão direta entre uma ou mais artérias coronárias e câmaras cardíacas ou um grande vaso; pode estar associada à cardiopatia congênita.

Objetivo: Estabelecer os padrões de trajetos de FAC a partir de dados ecocardiográficos e correlacioná-los com aspectos clínicos e cardiopatias congênitas.

Métodos: Um total de 7.183 prontuários médicos de crianças menores de 5 anos de idade com cardiopatia submetidas a ecodopplercardiograma colorido foram analisados utilizando o teste de correlação de Spearman para associar sinais, sintomas e cardiopatia à FAC, com nível de significância de $5 \%$.

Resultados: Vinte e seis crianças $(0,0036 \%)$ apresentaram FAC, nos seguintes trajetos: da artéria coronária direita para o ventrículo direito $(26,92 \%)$, da artéria coronária esquerda para o ventrículo direito $(23,08 \%)$, do ramo interventricular anterior para o ventrículo direito $(23,08 \%)$, da artéria coronária direita para o átrio direito $(11,54 \%)$, da artéria coronária esquerda para o tronco pulmonar $(7,69 \%)$ e do ramo interventricular anterior para o tronco pulmonar (7,69\%). Em $57,69 \%$ dos pacientes, houve uma correlação positiva entre sintomas e a FAC $(p=0,445)$, relacionada à dispneia ou cianose $(53,84 \%)$. Em $96,15 \%$, a cardiopatia congênita estava associada à FAC; principalmente, a comunicação interventricular e a comunicação interatrial, em 34,62\% dos casos, correlacionaram-se positivamente com a FAC $(p=0,295)$. O trajeto da FAC foi representado em três dimensões pelo software de modelagem, texturização e animação Cinema 4D R19.

Conclusão: A FAC é uma entidade anatômica incomum que apresenta quadro clínico compatível com dispneia e cianose e está associada a cardiopatias congênitas, principalmente com a CIV ou a CIA. De acordo com as análises ecocardiográficas, as fístulas na ACD, na ACE ou no RIVA representam aproximadamente um terço dos pacientes, com trajeto prioritário para as câmaras cardíacas direitas.

Palavras-chave: Doença da Artéria Coronária; Fístula Artério-Arterial; Cardiopatias Congênitas; Anatomia; Dispnea; Cianose; Ecocardiografia/métodos; Imagem Tridimensional/métodos.

\begin{abstract}
Background: Coronary artery fistula (CAF) is a direct connection of one or more coronary arteries to cardiac chambers or a large vessel; it may be associated with congenital heart disease.

Objective: To establish CAF pathway patterns from echocardiographic data and to correlate them with clinical aspects and congenital heart disease.

Methods: A total of 7.183 medical records of children under the age of five years with cardiac disease submitted to color Doppler echocardiography and Spearman's Correlation test were used to associate signs and symptoms and cardiopathy to CAF with a significance level of 5\%.

Results: Twenty six children (0.0036\%) presented CAF: from the right coronary artery (RCA) to the right ventricle (RV) $26.92 \%$, from the left coronary artery (LCA) to the RV 23.08\%, from the anterior interventricular branch (AIVB) to RV 23.08\%, RCA to right atrium (RA) $11.54 \%$, LCA for pulmonary trunk (PT) 7.69\% or AIVB for PT 7.69\%. In 57.69\% of the patients, there was a positively correlated symptomatology to CAF with $p=0.445$ related to dyspnea or cyanosis (53.84\%); in $96.15 \%$, congenital heart disease associated with CAF, mainly interventricular communication (IVC) or interatrial communication (IAC) in 34.62\% positively correlated to CAF with $p=0.295$. CAF pathway was represented in three dimensions by software modeling, texturing and animation Cinema 4D R19.
\end{abstract}

Correspondência: Daniel L. Cobo •

Faculdade de Medicina de São José do Rio Preto - Av. Brigadeiro Faria Lima, 5416. CEP 15090-000, Vila São Pedro, São José do Rio Preto, SP - Brasil E-mail: daniellcobo@gmail.com

Artigo recebido em 27/08/2019, revisado em 08/06/2020, aceito em 05/08/2020

DOI: https://doi.org/10.36660/abc.20190578 
Conclusion: CAF is an uncommon anatomical entity that presents a clinical picture compatible with dyspnea and cyanosis, and this is associated with congenital heart disease, mainly with IVC or IAC. According to echocardiographic analyzes, fistulas in RCA, LCA, or AIVB represent about one-third of the patients, with a priority pathway for right heart chambers.

Keywords: Coronary Artery Disease; Arterio-Arterial Fistula, Congenital Heart Diseases; Anatomy; Dyspnea; Cyanosis; Echocardiography/ methods; Imaging Three-Dimensional/methods.

Full texts in English - http://www.arquivosonline.com.br

\section{Introdução}

A fístula da artéria coronária (FAC) é uma conexão direta entre uma ou mais artérias coronárias a câmaras cardíacas ou a um grande vaso. É uma das anomalias da artéria coronária mais comuns, embora seja considerada rara na população em geral. ${ }^{1,2}$ Está presente em $0,002 \%$ da população e representa $0,4 \%$ de todas as malformações cardíacas.

O ecocardiograma Doppler tem sido indicado para avaliação de cardiopatias congênitas devido a sua versatilidade diagnóstica, disponibilidade, custo-efetividade e quantidade de informações morfofuncionais que fornece para o coração, ${ }^{5}$

Devido à raridade da FAC e à potencial contribuição da sua caracterização topográfica, o presente estudo visou determinar os padrões de trajetos da FAC a partir de dados ecocardiográficos e correlacioná-los com aspectos clínicos e cardiopatias congênitas. ${ }^{6,7}$

\section{Método}

Após a aprovação ética, foram considerados 7.183 prontuários eletrônicos de pacientes pediátricos com ou sem cardiopatia congênita do Serviço de Cardiologia e Cirurgia Cardiovascular Pediátrica do Hospital de Base e do Hospital da Criança e Maternidade de São José do Rio Preto, São Paulo, Brasil. Foram realizados exames ecocardiográficos bidimensionais em cores (Philips Healthcare ${ }^{\circledR}$ modelos HD 11 e HD 15) de acordo com as diretrizes da Sociedade Americana de Ecocardiografia. ${ }^{8}$ Crianças com outros defeitos cardíacos congênitos que não FAC e crianças com mais de cinco anos de idade devido a cardiopatia congênita sob cuidados durante os primeiros meses até os primeiros anos de vida foram excluídos do estudo. Foi definida por conveniência o tamanho da amostra do presente estudo.

\section{Análise estatística}

Foram obtidas as análises estatísticas utilizando o software SPSS Statistics versão $23.0^{9}$ e Excel (versão 2.016). As variáveis categóricas consideradas no presente estudo compreenderam os seguintes sinais e sintomas: assintomático, baixa saturação de $\mathrm{O}_{2^{\prime}}$ cianose (das extremidades, labial ou generalizada), hipertermia e dispneia; as seguintes cardiopatias associadas: atresia pulmonar, coarctação da aorta, comunicação interatrial $(\mathrm{ClA})$ ou comunicação interventricular (CIV), defeito do septo atrioventricular, estenose da válvula pulmonar, ducto arterial persistente, tetralogia de Fallot e sem cardiopatia associada; bem como a topografia da FAC a partir da descrição de relatórios ecocardiográficos para reconstrução tridimensional por pelo software de modelagem, texturização e animação Cinema 4D R19. ${ }^{10}$ A normalidade dos dados foi verificada por meio do teste de Shapiro-Wilk, que apresentou dados não paramétricos. Posteriormente, foi aplicado o teste de correlação de Spearman para associar as cardiopatias congênitas e os sinais e sintomas com a FAC, estabelecendo valor de $<0,05 .{ }^{9}$

\section{Resultados}

No presente estudo, foi apresentada uma análise descritiva (Tabela 1) e, para as variáveis categóricas, foi analisado o cruzamento inferencial (Tabelas 2 e 3). Foi utilizado o teste de correlação de Spearman e foi apresentado um único valor de p para cada cruzamento. Dos 7.183 prontuários considerados, foi detectada a FAC em 26 casos (0,0036\%). A Tabela 1 mostra as variáveis categóricas de trajetos de FAC detectados por ecocardiograma, demonstrando que as fístulas na artéria coronária direita (ACD), na artéria coronária esquerda (ACE) ou no ramo interventricular anterior (RIVA) representaram aproximadamente um terço dos pacientes, com trajeto prioritário para as câmaras cardíacas direitas.

A Tabela 2 correlacionou as variáveis categóricas, os sinais e sintomas (assintomáticos, baixa saturação de $\mathrm{O}_{2}$, cianose das extremidades, labial ou generalizada, hipertermia e dispneia) com os tipos da FAC (ACD, ventrículo direito, átrio direito, $\mathrm{ACE}$, tronco pulmonar e RIVA) e mostra que $26,92 \%$ dos pacientes com FAC eram assintomáticos e 57,69\% eram sintomáticos, expressos por dispneia (26,92\%), cianose (26,92\%) e hipertermia (3,86\%). A baixa saturação de oxigênio foi detectada pelo oxímetro de pulso em $15,38 \%$ dos pacientes. A partir desses dados, foi realizado o teste de correlação de Spearman, que observou valor de $p=0,445$, demonstrando não haver evidência estatística de dependência entre as variáveis analisadas.

A Tabela 3 mostra que a FAC esteve associada à cardiopatia congênita em 96,15\% dos casos, principalmente com a CIV ou a ClA em 34,62\% dos casos, a tetralogia de Fallot (23,08\%)

Tabela 1 - Variáveis categóricas de trajetos de fístula da artéria coronária detectados por ecocardiograma

\begin{tabular}{lcc}
\hline Tipo de fistula & $\mathbf{N}$ & $\%$ \\
\hline ACD para VD & 7 & 26,92 \\
\hline ACD para AD & 3 & 11,54 \\
\hline ACE para TP & 2 & 7,69 \\
\hline ACE para VD & 6 & 23,08 \\
\hline RIVA para TP & 2 & 7,69 \\
\hline RIVA para VD & 6 & 23,08 \\
\hline Total & $\mathbf{2 6}$ & $\mathbf{1 0 0}$ \\
\hline $\begin{array}{l}\text { ACD: artéria coronária direita, ACE: artéria coronária esquerda; } A D: \\
\text { átrio direito; N: valor absoluto; RIVA: ramo interventricular anterior; } \\
\text { TP: tronco pulmonar; VD: ventrículo direito. }\end{array}$ & &
\end{tabular}


Tabela 2 - Correlação das variáveis categóricas de sinais e sintomas com os tipos de fístula da artéria coronária

\begin{tabular}{|c|c|c|c|c|c|c|c|c|c|c|c|c|c|c|}
\hline \multirow{3}{*}{ Sinais e sintomas } & \multicolumn{12}{|c|}{ Tipos de fístula da artéria coronária } & \multirow{2}{*}{\multicolumn{2}{|c|}{ TOTAL }} \\
\hline & \multicolumn{2}{|c|}{ ACD/VD } & \multicolumn{2}{|c|}{ ACD/AD } & \multicolumn{2}{|c|}{ ACE/TP } & \multicolumn{2}{|c|}{ ACE/VD } & \multicolumn{2}{|c|}{ RIVA/TP } & \multicolumn{2}{|c|}{ RIVA/VD } & & \\
\hline & N & $\%$ & N & $\%$ & N & $\%$ & N & $\%$ & N & $\%$ & $\mathbf{N}$ & $\%$ & N & $\%$ \\
\hline Assintomático & 0 & 0 & 2 & 66,67 & 1 & 50 & 2 & 33,33 & 0 & 0 & 2 & 33,33 & 7 & 26,92 \\
\hline Baixa saturação de $\mathrm{O}_{2}$ & 2 & 28,57 & 0 & 0 & 0 & 0 & 2 & 33,33 & 0 & 0 & 0 & 0 & 4 & 15,38 \\
\hline $\begin{array}{l}\text { Cianose das extremidades, } \\
\text { labial ou generalizada }\end{array}$ & 3 & 42,86 & 0 & 0 & 0 & 0 & 0 & 0 & 1 & 50 & 3 & 50 & 7 & 26,92 \\
\hline Hipertermia & 0 & 0 & 0 & 0 & 0 & 0 & 0 & 0 & 0 & 0 & 1 & 16,67 & 1 & 3,86 \\
\hline Dispneia & 2 & 28,57 & 1 & 33,33 & 1 & 50 & 2 & 33,33 & 1 & 50 & 0 & 0 & 7 & 26,92 \\
\hline Total & 7 & 100 & 3 & 100 & 2 & 100 & 6 & 100 & 2 & 100 & 6 & 100 & 26 & 100 \\
\hline Valor (p) & & & & & & & & & & & & & & ,445 \\
\hline
\end{tabular}

ACD: artéria coronária direita; $A C E$ : artéria coronária esquerda; $A D$ : átrio direito; N: valor absoluto; RIVA: ramo interventricular anterior; TP: tronco pulmonar; VD: ventrículo direito.

Tabela 3 - Correlação das variáveis categóricas de cardiopatia associada com os tipos de fístula da artéria coronária

\begin{tabular}{|c|c|c|c|c|c|c|c|c|c|c|c|c|c|c|}
\hline \multirow{3}{*}{ Doença cardíaca associada } & \multicolumn{12}{|c|}{ Tipos de fístula da artéria coronária } & \multirow{2}{*}{\multicolumn{2}{|c|}{ TOTAL }} \\
\hline & \multicolumn{2}{|c|}{ ACD/VD } & \multicolumn{2}{|c|}{ ACD/AD } & \multicolumn{2}{|c|}{ ACE/TP } & \multicolumn{2}{|c|}{ ACE/VD } & \multicolumn{2}{|c|}{ RIVA/TP } & \multicolumn{2}{|c|}{ RIVA/VD } & & \\
\hline & $\mathbf{N}$ & $\%$ & $\mathbf{N}$ & $\%$ & $\mathbf{N}$ & $\%$ & $\mathbf{N}$ & $\%$ & $\mathbf{N}$ & $\%$ & N & $\%$ & $\mathbf{N}$ & $\%$ \\
\hline Atresia pulmonar & 1 & 14,29 & 0 & 0 & 0 & 0 & 0 & 0 & 0 & 0 & 0 & 0 & 1 & 3,85 \\
\hline Coarctação da aorta & 0 & 0 & 0 & 0 & 1 & 50 & 0 & 0 & 1 & 50 & 1 & 16,67 & 3 & 11,53 \\
\hline CIA ou CIV & 2 & 28,57 & 2 & 66,67 & 0 & 0 & 2 & 33,33 & 0 & 0 & 3 & 50 & 9 & 34,62 \\
\hline Defeito do septo atrioventricular & 0 & 0 & 0 & 0 & 0 & 0 & 0 & 0 & 0 & 0 & 1 & 16,67 & 1 & 3,85 \\
\hline Estenose da válvula pulmonar & 1 & 14,29 & 0 & 0 & 0 & 0 & 0 & 0 & 0 & 0 & 1 & 16,67 & 2 & 7,69 \\
\hline Sem cardiopatia associada & 0 & 0 & 1 & 33,33 & 1 & 50 & 1 & 16,67 & 0 & 0 & 0 & 0 & 3 & 11,53 \\
\hline Ducto arterioso persistente & 0 & 0 & 0 & 0 & 0 & 0 & 0 & 0 & 1 & 50 & 0 & 0 & 1 & 3,85 \\
\hline Tetralogia de Fallot & 3 & 42,86 & 0 & 0 & 0 & 0 & 3 & 50 & 0 & 0 & 0 & 0 & 6 & 23,08 \\
\hline Total & 7 & 100 & 3 & 100 & 2 & 100 & 6 & 100 & 2 & 100 & 6 & 100 & 26 & 100 \\
\hline Valor (p) & & & & & & & & & & & & & 0,29 & \\
\hline
\end{tabular}

$A C D$ : artéria coronária direita; $A C E$ : artéria coronária esquerda; $A D$ : átrio direito; CIA: comunicação interatrial; CIV: comunicação interventricular; N: valor absoluto; RIVA: ramo interventricular anterior; TP: tronco pulmonar; VD: ventrículo direito.

e a coarctação de aorta (11,53\%). As variáveis categóricas de cardiopatia associada (atresia pulmonar, coarctação da aorta, CIA ou CIV, defeito do septo atrioventricular, estenose da válvula pulmonar, ducto arterioso persistente, tetralogia de Fallot e sem cardiopatia associada) foram correlacionadas com os tipos de FAC (ACD, ventrículo direito, átrio direito, $\mathrm{ACE}$, tronco pulmonar e RIVA) e, a partir desses dados, foi realizado o teste de correlação de Spearman, que observou valor de $p=0,295$, demonstrando não haver evidência estatística de dependência entre as variáveis analisadas.

A Figura 1 mostra os padrões de trajeto encontrados para a FAC e os seus percentuais respectivos. ${ }^{10}$

\section{Discussão}

No presente estudo, a prevalência de FAC foi observada em $0,0036 \%$ dos casos, embora a frequência real de anomalias da artéria coronária na população geral possa ser desconhecida ou extremamente rara. ${ }^{11}$ A prevalência estimada varia de $0,002 \%{ }^{12}$ a
$0,1 \%$ ou de $1 \%$ a $2 \%,{ }^{13,14}$ e representa $14 \%$ de todas as anomalias da artéria coronária. ${ }^{15}$

Quase um terço dos pacientes eram assintomáticos; os sinais e sintomas foram representados por dispneia, cianose e hipertermia em 57,69\% deles (Tabela 2). Embora a ausência de sintomas possa ser ainda mais prevalente, a angina devido ao fenômeno de "roubo coronário" (débito cardíaco diminuído), a endocardite, o infarto agudo do miocárdio ou a insuficiência cardíaca podem estar presentes. ${ }^{14,16}$ As crianças com FAC são frequentemente assintomáticas; portanto, estima-se que aproximadamente $80 \%$ dos pacientes menores de 20 anos de idade sejam assintomáticos. ${ }^{17}$

No presente estudo, a maioria das crianças $(96,15 \%)$ com FAC apresentava outra cardiopatia congênita associada, principalmente CIV ou CIA, em um terço dos pacientes (Tabela 3). No entanto, a FAC como uma manifestação isolada pode estar presente em 55\% a 80\% dos casos e foi associada à cardiopatia congênita (tetralogia de Fallot, ducto arterioso persistente, CIV ou CIA) em $20 \%$ a $45 \%{ }^{17}$ e doença arterial coronariana em até 
Artéria coronária esquerda para ventrículo direito $(23,08 \%)$

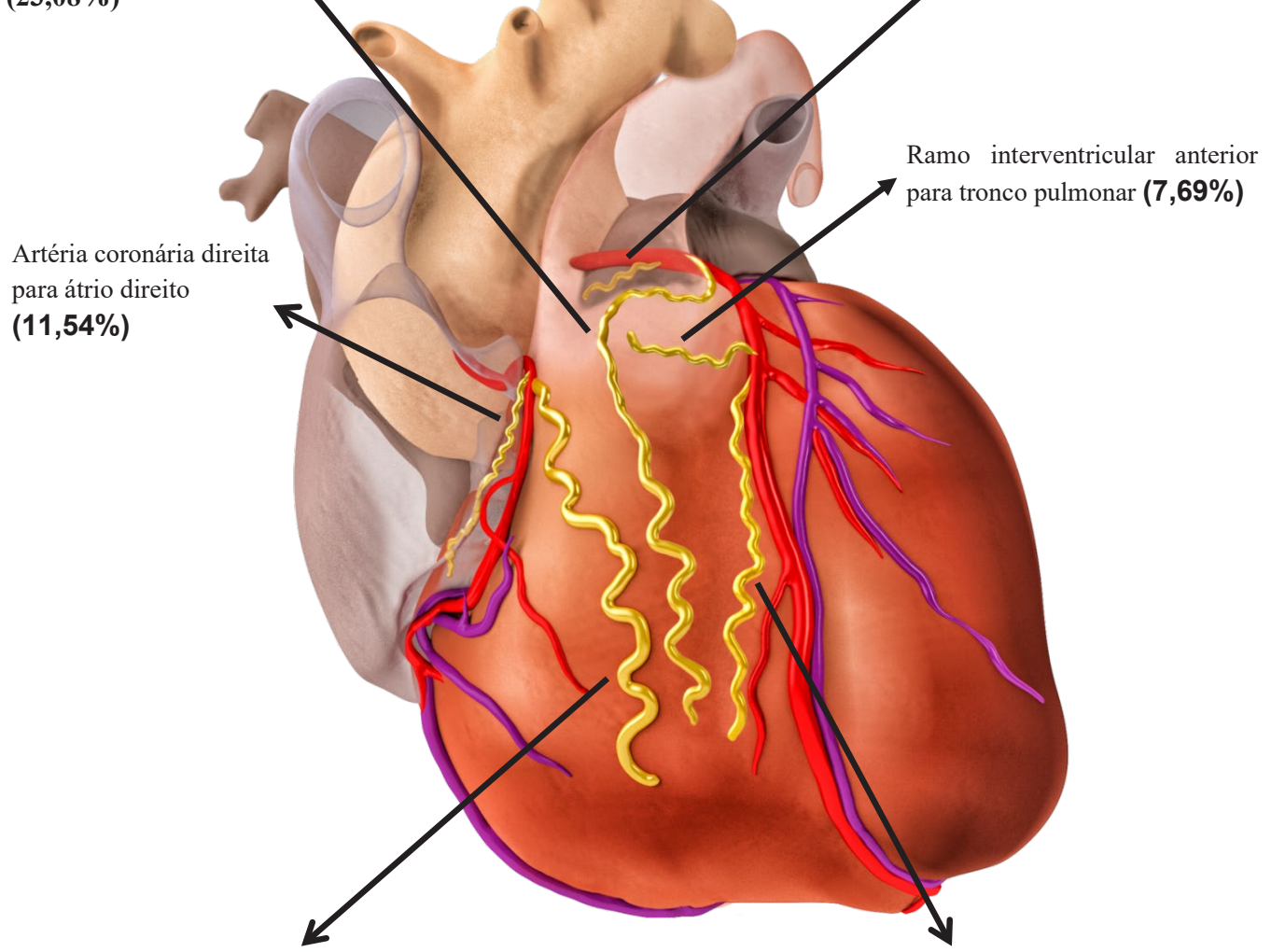

Artéria coronária direita para ventrículo direito $(\mathbf{2 6 , 9 2 \% )}$

Ramo interventricular anterior para ventrículo direito $(\mathbf{2 3 , 0 8 \% )}$

Figura 1 - Representação topografia de trajetos de fistula da artéria coronária e seus percentuais respectivos.

$35 \%$ das ocorrências. ${ }^{18}$ Além disso, a FAC frequentemente tem etiologia congênita, mas pode ser secundária a lesão, infecção, causas iatrogênicas ou doença de Kawasaki. ${ }^{12}$

De acordo com as análises ecocardiográficas, os resultados destacaram que as fístulas na ACD, na ACE ou no RIVA representam aproximadamente um terço dos pacientes, com trajeto prioritário para as câmaras cardíacas direitas (Tabela 1). Manoly et al., relataram que a FAC é mais prevalente na ACD (52\% dos casos avaliados), com drenagem para o lado direito do coração em mais de $90 \%$ dos casos, o que foi corroborado por outros autores. ${ }^{16}$

A ecocardiografia bidimensional colorida tem sido recomendada para avaliar a FAC, embora a angiografia, ${ }^{1,16}$ o ecocardiograma transtorácico e a angiotomografia computadorizada apresentem resultados eficazes. ${ }^{2,19}$ Uma possível limitação do presente estudo refere-se à maior prevalência da FAC na $\mathrm{ACD}$ do que no átrio direito, possivelmente decorrente da forma iatrogênica após procedimento cirúrgico para correção da tetralogia de Fallot. 20,21

Devido à baixa prevalência da FAC, está disponível um número reduzido de publicações científicas sobre o assunto. A contribuição do presente estudo aponta para o tamanho relativamente grande da amostra (26 registros) para uma anomalia cardíaca rara. ${ }^{1,4}$
Enquanto as análises ecocardiográficas nos prontuários eletrônicos apresentam representação bidimensional, a análise tridimensional realizada neste estudo pode ser mais eficaz. ${ }^{22}$ Os esforços para melhorar a imagem para FAC, de preferência tridimensional, podem melhorar o tratamento clínico, o planejamento cirúrgico e a intervenção intraoperatória. ${ }^{23}$

\section{Conclusões}

A FAC é uma entidade anatômica incomum que apresenta quadro clínico compatível com dispneia e cianose e está associada a cardiopatias congênitas, principalmente com a CIV ou a CIA. De acordo com as análises ecocardiográficas, as fístulas na ACD, na ACE ou no RIVA estão presentes em aproximadamente um terço dos pacientes, com trajeto prioritário para as câmaras cardíacas direitas.

\section{Agradecimentos}

Agradeço ao meu orientador Fernando Batigália pela excelente condução deste trabalho. Agradeço também a Ulisses A. Croti pela autorização do estudo no setor de Cirurgia Cardiovascular Pediátrica do Hospital Infantil e Maternidade e Adília M. P. 
Sciarra, Marcos H.D. Foss e Rafaela G.F. Cobo pelo auxílio na correção e tradução do manuscrito.

\section{Contribuição dos autores}

Concepção e desenho da pesquisa e Análise e interpretação dos dados: Cobo DL, Batigalia F, Croti UA; Obtenção de dados: Cobo DL, Batigalia F, Croti UA, Cobo RGF; Análise estatística: Cobo DL, Batigalia F; Obtenção de financiamento: Cobo DL; Redação do manuscrito e Revisão crítica do manuscrito quanto ao conteúdo intelectual importante: Cobo DL, Batigalia F, Croti UA, Sciarra AMP, Foss MHD, Cobo RGF.

\section{Potencial conflito de interesse}

Não há conflito com o presente artigo

\section{Referências}

1. Challoumas D, Pericleous A, Dimitrakaki IA, Danelatos C, Dimitrakakis G. Coronary Arteriovenous Fistulae: A Review. Int J Angiol.2014 Mar;23(1):1-10.

2. Lee DY, Park SH, Bae MH, Lee JH, Yang DG, Park HS, et al. Multiple Fistula Emptying into the Left Ventricle Through the Entire Left Ventricular Wall. J Cardiovasc Ultrasound. 2012 Jun;20(2):108-11.

3. Loukas M, Germain AS, Gabriel A, John A, Tubbs RS, Spicer D. Coronary Artery Fistula: A Review. Cardiovasc Pathol. May-Jun 2015;24(3):141-8.

4. Xie M, Li L, Cheng TO, Sun Z, Wang X, Lv Q. et al. Coronary Artery Fistula: Comparison of Diagnostic Accuracy by Echocardiography versus Coronary Arteriography and Surgery in 63 Patients Studied between 2002 and 2012 in a Single Medical Center in China. Int J Cardiol. 2014 Sep 20;176(2):470-7.

5. Almeida I, Caetano F, Trigo J, Mota P,Marques AL. Transthoracic Echocardiography in the Diagnosis of Coronary Fistula. Rev Port Cardiol. 2014 Oct;33(10):655-6.

6. Delgado A, Moreira D, Rodrigues B, Correia E, Gama P, Cabral C. et al. Hypertrophic cardiomyopathy associated with left ventricular noncompaction cardiomyopathy and coronary fistulae: A case report. One genotype, three phenotypes?. Rev Port Cardiol. 2013 Nov;32(11):919-24.

7. Manoly I, Mahadevan VS, Hoschtitzky JA. Hybrid Approach to Closure of an Acquired Coronary-Cameral Fistula. Ann Thorac Surg. 2014 Sep;98(3):e59-61.

8. Lang RM, Badano LP, Mor-avi V, Afilalo J, Armstrong A, Ernande L, et al. Recommendations for Cardiac Chamber Quantification by Echocardiography in Adults: An Update from the American Society of Echocardiography and the European Association of Cardiovascular Imaging. Guidelines and Standards. J Am Soc Echocardiogr. 2015 Jan;28(1):1-39.e14

9. Marôco J. Análise Estatística com o SPSS Statistics. 7 ed. Portugal: Pêro Pinheiro - Portugal: Gráfica Manoel Barbosa \& Filhos; 2018. p. 657-780.

10. Maxon a Nemetschek Company. Cinema 4D R19, versão 2018. [Cited in 2018 Jul 28]. Available from: < https://www.maxon.net/en-gb/products/cinema-4d/ overview>

11. Haddad J, Novaes G, Schimidt A, Oliveira M, Figueiredo G, Marin Neto JA. Rare form of coronary fistula draining into the right ventricular apex. Rev Bras Cardiol. 2016; 24(1-4): 44-6.

12. Valera FJ, Doñate L, Hernández CE, Schuler M, Bel A, Montero JA. Surgical treatment of circumflex artery aneurysm with fistula to the coronary sinus and aortic regurgitation. Cir Cardiov. 2014; 21(1):60-2.

\section{Fontes de financiamento}

O presente estudo não teve fontes de financiamento externas.

\section{Vinculação acadêmica}

Este artigo é parte de dissertação de mestrado de Daniel L. Cobo pela Faculdade de Medicina de São José do Rio Preto-FAMERP-SP.

\section{Aprovação ética e consentimento informado}

Este estudo foi aprovado pelo Comitê de Ética da Faculdade de Medicina de São José do Rio Preto-FAMERPSP sob o número de protocolo 81217417.7.0000.54. Todos os procedimentos envolvidos nesse estudo estão de acordo com a Declaração de Helsinki de 1975, atualizada em 2013.

13. Domínguez-Massa C, Bel-Mínguez AM, Pérez-Guillén M, Valera-Martínez FJ, Hornero-Sos F. Coronary fistula from circumflex artery to superior caval vein. Cir. Cardiov. 2018; 25(3):170-2.

14. Ospina-Galeano AM, Navas-Gutiérrez SE, Ospina-Galeano DC, Bautista H, Velandia-Carrillo C. Anomalous Origin of the Right Coronary Artery With an Interarterial Course. Rev Colomb. Cardiol. 2017; 24(2):128.

15. Buccheri D, Chirco PR, Geraci S, Caramanno G, Cortese B. Coronary Artery Fistulae: Anatomy, Diagnosis and ManagementStrategies. Heart Lung Circ. 2018; 27(8):940-51.

16. Van Caenegem M, Vandekerckhove H. Coronary pulmonary fistula: A case series. Int J Case Rep Imag. 2016; 7(5):292-5.

17. Buccheri D, Luparelli M, Chirco PR, Piraino D, Andolina G, Assennato P. A call to action for an underestimated entity: Our algorithm for diagnosis and management of coronary artery fistula. Int J Cardiol. 2016 Oct 15; 221:1081-3.

18. Contreras-Gutiérrez VH, Téllez-Ramírez RA. Coronary arterio-venous fistula associated acute coronary syndrome: A case-report and review of literature. Rev Med Hosp Gen Méx. 2017;80(1):51-5.

19. Kline AD, Gupta D, Mastin ST, Chadran A. Coronary Cardiac Fistula: Evaluation by Cardiac CTA for Management. World J Pediatr Congenit Heart Surg. 2015 Jul;6(3):484-5.

20. Leardini P, Piotto VR, Silva MS, Tjeng R. Coronary Fistula. Perspectivas Médicas: Red. de Revistas Científicas de América Latina y el Caribe, España y Portugal. $2006 ; 17: 26-8$

21. Mulinari LA, Navarro, FB, Pimentel GK, Miyazaki SM, Binotto CN, Pelissari EC. et al. The use and midium-term evaluation of decellularized allograft cusp in the surgical treatment of the tetralogy of Fallot. Rev Bras Cir Cardiovas. 2008; 23(2):197-203.

22. Joshi JK, Beache GM, Slaughter MS, Sobieski MA, Schneider W, Stoddard MF. Coronary Artery Fistula: 64-Slice Computed Tomographic Delineation and Correlation with Multiplane Transesophageal Echocardiography and Surgical Findings. Echocardiography. 2012; 29(3):69-71.

23. Attili A, Hensley AK, Jones FD, Grabham J, Dissesa TG. Echocardiography and Coronary CT Angiography Imaging of Variations in Coronary Anatomy and Coronary Abnormalities in Athletic Children: Detection of Coronary Abnormalities that Create a Risk for Sudden Death. Echocardiography. 2013; 30(2): 225-33. 